To the Editors:

\title{
Real time surveillance of dengue fever - a preliminary study
}

\author{
Y P J N Warnasekara', S B Agampodi', P M N C Marage', A A S D Athurupana', S D Koonthota', \\ N J Dahanayaka ${ }^{1}$
}

Ceylon Medical Journal 2016; 61: 197-198

http://doi.org/10.4038/cmj.v61i4.8393

Dengue is the commonest infectious disease in Sri Lanka. It is a notifiable disease since 1996. There were 32063 dengue fever patients reported from Sri Lanka in 2013 and most of them were from urban and semi urban areas [1]. Dengue surveillance programme is one of the most important components of dengue control and prevention. Sri Lanka has a well-established and functioning notification system for communicable diseases surveillance. This is a paper based system and the notification forms which are filled by the first contact medical officer are sent to the range Public Health Inspector (PHI) for preventive actions through a process involving Infectious Controlling Nursing Officers (ICNO), head of the institutions and Medical Officer of Health (MOH). Since this involves the usual postal system and several individuals, this process might take even up to one to two weeks to reach PHI which may be too late for early intervention [2]. This maybe further delayed when patients acquire dengue while away from home and are subsequently admitted to a hospitals close to their home.

Mobile technology in disease surveillance has been used for surveillance of numerous health conditions [3,5]. However, mobile technology is not used in Sri Lanka for disease control measures and only few studies have been conducted on this [6,7]. Digital mapping and Global Positioning System (GPS) are available in most smart phones, and is freely available throughout the country. The objective of our study was to field test the feasibility of digital mapping using locus free mapping rather than going to field and the use of real time surveillance for dengue in Anuradhapura. Approval was obtained from Ethics Review Committee, Faculty of Medicine and Allied Sciences, Rajarata University of Sri Lanka.

A descriptive cross sectional study was conducted as a real time spatial temporal monitoring of the probable acquiring sites of the suspected dengue patients admitted to all three medical units of Teaching Hospital Anuradhapura (THA) from 29 September 2013 to 2 February 2014. A medical officer visited all three medical wards daily and interviewed the patients for approximately five minutes regarding the onset of disease, environmental factors, occupation and travel history during incubation period with the aim of identifying the most probable sites of acquiring the disease. Then the probable sites of acquiring dengue were located on an online map using locus free software by showing the locations of the map to the patient using a low cost tablet computer. Special emphasis was given to home, work place and places visited recently. Different flags marked the location of the home, work place and the most probable site of acquiring the disease. If there were no cases reported from his or her home or work place, patient was questioned about places visited with special emphasis on areas from where dengue cases have already been reported.

While conducting the survey, the investigating team carefully looked for clustering of cases within the NCP (indigenous cases) and outside the NCP. A potential indigenous cluster was defined as two cases reported within a period of one week, where the probable disease acquired sites were situated within a radius of $100 \mathrm{~m}$, without a travel history outside the NCP. A cluster outside the NCP was defined as two cases reported within a period of one week where the probable disease acquired sites within a radius of $100 \mathrm{~m}$ and were outside the NCP.

There were 180 patients with a clinical diagnosis dengue during the study period. Majority of them (132) were males and mean age was 40 (SD 18) years. The response rate was $100 \%$, as everybody included in the study consented because the interview was short and simple. Out of 180 suspected patients, 35 had not travelled beyond the Anuradhapura District within the incubation period. We identified clustering of cases in Bank Town Anuradhapura (4 cases), Nikawewa ( 3 cases), and Thalawa ( 2 cases) as potential indigenous dengue transmission sites. Thalawa cluster had two patients living in the area. In Bank Town, Anuradhapura three patients were working in adjacent shops and a fourth patient used the grounds for exercise. No dengue cases were reported

${ }^{1}$ Faculty of Medicine and Allied Sciences, Rajarata University of Sri Lanka.

Correspondence: YPJNW, e-mail: <jwarnasekara@yahoo.com>. Received 28 April 2016.

This is an open-access article distributed under the terms of the Creative Commons Attribution License, which permits unrestricted use, distribution, and reproduction in any medium, provided the original author and source are credited. 
from homes or work places. Nikawewa cluster consisted of one patient from the area and two bus drivers who park their buses at the Nikawewa junction. The Regional Epidemiologist (RE) was informed immediately after the detection of a second patient from each area. There were two further cases from Bank Town Anuradhapura and one case from Thalawa. No cases were reported from the Nikawewa cluster during the period of study.

There were 135 patients who stayed in or visited areas outside the Anuradhapura District during the incubation period. Of them, 120 had visited Colombo. Obvious clustering of cases were noted in Narahenpita, close to Sugathadasa stadium, Wellawatta, Colombo 3 and Dematagoda and most of these places were construction sites. The patients were construction workers whose residence was situated in NCP but had lived outside the NCP during the incubation period.

This study provides preliminary evidence to suggest that digital mapping and real time surveillance of dengue is feasible and provides rapid results, compared to the current paper based notification system. The popular use of social media and internet based software is a factor which supports the feasibility of establishing such a system in Sri Lanka. A system where infection controlling nurses carry mobile tablets rather than notification form, real-time update of dengue cases on online maps, which are shared with $\mathrm{MOH}$ and RE will provide a rapid system to identify clustering of dengue and indigenous transmission. This will provide quick action to minimise spread of dengue. It will also allow the general public to have access to data on areas affected by dengue.
We acknowledge the Regional Epidemiologist, Anuradhapura for initiating dengue prevention based on our data.

\section{References}

1. Sri Lankan Situation. http://www.dengue.health.gov.lk/ index.php/information-on-dengue/sri-lankan-situation. Accessed December 23, 2015.

2. Seneviratne SL, Gunatilake SB, de Silva HJ. Reporting notifiable diseases: methods for improvement, attitudes and community outcome. Trans R Soc Trop Med Hyg 1997; 91: 135-7.

3.. Robertson C, Sawford K, Daniel SLA, Nelson TA, Stephen C. Mobile phone-based infectious disease surveillance system, Sri Lanka. Emerg Infect Dis 2010; 16: 1524-31.

4. Yang C, Yang J, Luo X, Gong P. Use of mobile phones in an emergency reporting system for infectious disease surveillance after the Sichuan earthquake in China. Bull World Health Organ 2009; 87: 619-23.

5. Brownstein J. Digital disease detection-harnessing the Web for public health surveillance. New Engl J Med 2009; 360: 2153-5.

6. Lwin MO, Vijaykumar S, Lim G, Fernando ONN, Rathnayake VS, Foo S. Baseline Evaluation of a Participatory Mobile Health Intervention for Dengue Prevention in Sri Lanka. Health Educ Behav 2016; 43: 471-4.

7. Lwin MO, Vijaykumar S, Fernando ONN, et al. A $21^{\text {st }}$ century approach to tackling dengue: Crowdsourced surveillance, predictive mapping and tailored communication. Acta Trop 2014; 130: 100-7. 